\title{
Working with Generative Systems: An Artistic Perspective
}

\author{
Jon McCormack \\ sensiLab, Monash University \\ Caulfield East, Australia \\ Jon.McCormack@monash.edu
}

\begin{abstract}
Generative systems are unique in that they allow the artist to specify processes rather than outcomes. In this paper, I describe my creative approach to working with complex computational systems that I have designed and coded over several decades. These systems require certain ways of working that offer both liberation and restriction on creative practice. To illustrate how these systems work in a creative sense, I will give an overview of two recent works: Fifty Sisters (20122016) and The Unknowable (2015-2017). Fifty Sisters comprises of fifty $1 \mathrm{~m} \times 1 \mathrm{~m}$ images of computer synthesised plant-forms, algorithmically "grown" from computer code using artificial evolution and generative grammars. Each plant-like form is derived from the primitive graphic elements of oil company logos. The title of the work refers to the original "Seven Sisters" - a cartel of seven oil companies that dominated the global petrochemical industry and Middle East oil production from the mid-1940s until the oil crisis of the 1970s. The Unknowable is a three-channel 4k video triptych. The work depicts a series of virtual flora, evolved using computer programs based on biological evolution. In each sequence evolved plant species slowly disintegrate and then reform, breaking into their fundamental component - the polygon - then reforming anew in an endless cycle of destruction and reconstitution. Both works are mediations on our changed relationship to nature and the natural in a globalised, disconnected virtual world now effectively navigated through digital media.
\end{abstract}

Generative art. Procedural modelling. L-systems. Evolution. Grammars. Computational sublime, Agency, Autonomy.

\section{INTRODUCTION}

I have worked with generative computational systems since the late 1980s. My interest in them arose from a fascination with the idea of using computers to compose virtual space and time with a conceptual freedom unmatched by any other human tools. Generative systems seemed like an ideal processual technique to most effectively explore this new terrain.

At that time, there was a significant interest in these systems from several science and technology disciplines, including computer graphics, artificial life, cybernetics and computational biology. Chris Langton's seminal paper introducing Artificial Life (Langton 1995) drew together several previously separate research strands to define a new field that sought to re-conceptualise life itself. For me it inspired many rich ideas that I have investigated in my work, illustrating that computational thinking can profoundly challenge and illuminate how we conceptualise the world.
In this paper, I will provide some insight into the design and development of two recent works and try to explain why I continue to find working with generative systems intellectually fascinating and creatively rewarding.

After describing the works and the processes used to develop them, I then briefly discuss what I consider to be important challenges and critiques raised by generative systems and artworks that make use of them (generative art).

\subsection{Generative Systems}

Generative systems are fundamentally concerned with process. As the name implies, this process causes the creation of something. Typically, generative systems are characterised by what Alvy Ray Smith called "database amplification" (Smith 1984), the idea that you can get more out of a generative system than you put into it. Central to this concept is the breaking of one of the so-called "Dictums of Descartes" - that a human design cannot outperform the designer's specification (Ashby 1952). 
Generative processes often exhibit emergent properties not explicitly stated in the process specification (McCormack \& Dorin 2001). They have the capacity for creating complexity, surprise and novelty. Generative art, which makes use of generative systems for artistic applications, is characterised by this approach rather than by any specific media, content or realisation.

In the case of my own work (and that of many others), the computer is an ideal medium for working with generative systems, since it is a flexible, programmable, process generating machine.

\section{FIFTY SISTERS}

Fifty Sisters is a generative artwork commissioned for the Ars Electronia museum in Linz, Austria in 2012. The work consists of 50 different digital images, inkjet-printed and mounted on aluminium composite panels, each $1 \mathrm{~m} \times 1 \mathrm{~m}$. Each image depicts a computer synthesised plant form, derived from the primitive graphic elements of oil company logos.

The title of the work refers to the original "Seven Sisters" - a cartel of seven oil companies that dominated the global petrochemical industry and Middle East oil production from the mid-1940s until the oil crisis of the 1970s. Fossil fuels began as plants that over millions of years were transformed by geological processes into the coal and oil that drives modern civilisation. By connecting the power of oil (both in terms of global finance and energy) back to its natural origins, the work reminds us of our technological reliance on a non-renewable resource. The clean graphic beauty of oil company logos - which often refer to plants - are repurposed from their benign origins and mutated into technoorganic hybrids, juxtaposing the mathematical beauty of nature with its innate gnarly complexity (Figure 1).

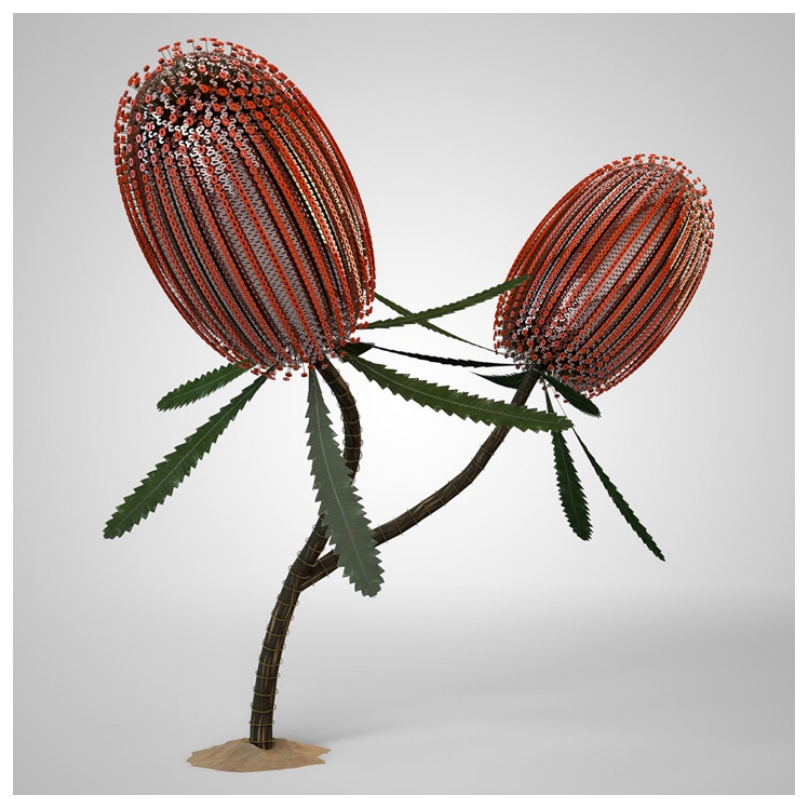

Figure 1: One of the Fifty Sisters. A Banksia-like species built from elements of the ESSO Petroleum logo.

The work was created using custom software I have developed since 1990, originally on Silicon Graphics computers, but now on Unix-based systems (including MacOS). The software uses a rule-based developmental model, loosely based on biological morphogenesis and derived from earlier work in developmental grammars such as Lsystems (Prusinkiewicz \& Lindenmayer 1990). Technical details can be found in (McCormack 2005). In simple terms, the software simulates the growth and development of the form from a series of developmental rules, metaphorically like the way DNA "encodes" the developmental plans of biological organisms. I refer to the developmental rules as "digital genes" in this paper.

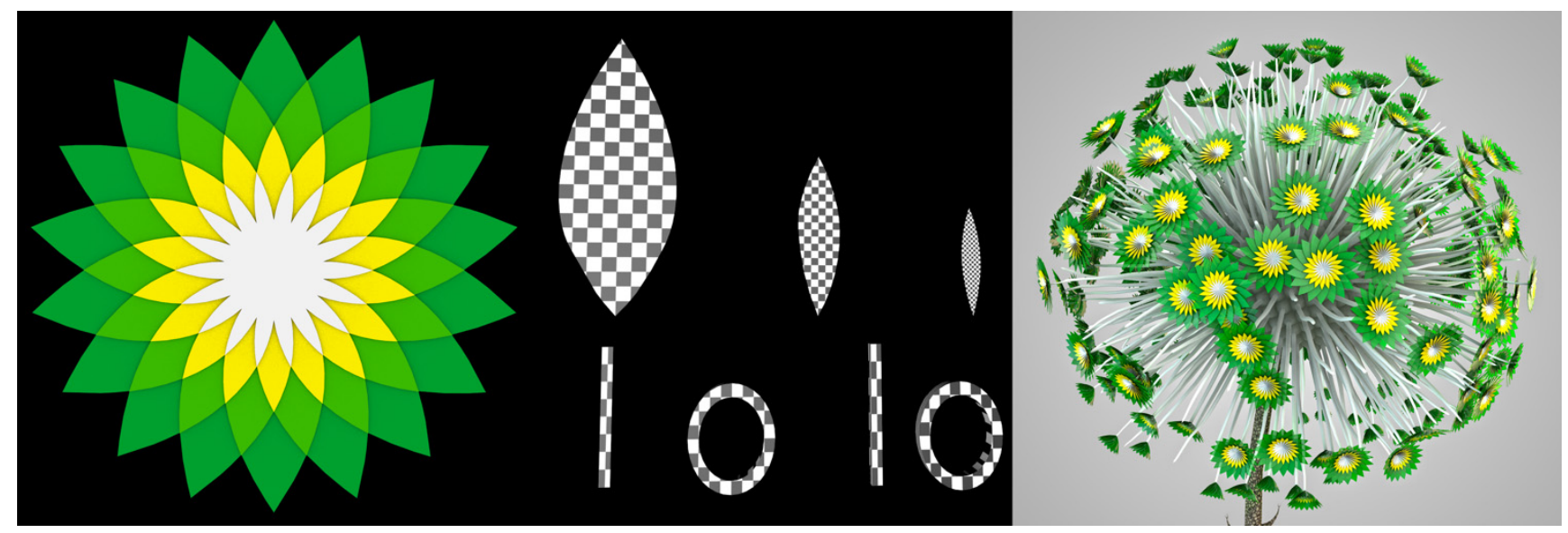

Figure 2: Stages of development: $3 D$ version of the BP logo (left), breakdown into graphic primitives (middle) and final evolved digital image built from the primitives (right). In this example the original logo is visible in many places in the final image, however various new structures have also emerged from the generative process. 
Developing the work involved several stages: (i) sourcing each original oil company logo; (ii) breaking down each logo into basic graphic primitives; (iii) converting each primitive to 3D geometry; (iv) evolving and "gene splicing" the genetic codes that define the growth and development of each unique plant form; (v) final rendering and output of each image. Figure 2 shows an example of some of these steps for one of the 50 forms.

Creating the developmental rules that define each plant form (step iv) encompasses first "reverse engineering" the developmental structure of known plant forms - a task done by hand, based largely on intuition and experience after many years of working with the generative system. Sets of developmental rules form a "gene pool" from which two further design processes occur: gene splicing where selected genes from different original forms are mixed together to create new hybrid forms, and interactive evolution where new forms a selectively bred using a process of rule mutation and selection (McCormack 1993).

These "genetic" manipulation processes produce many intermediate forms that are discarded along the way to the final form. Like the evolution of life on Earth, some branches of the phylogenetic tree are evolutionary dead-ends (although here for aesthetic or structural reasons). The decision to stop is based on a personal aesthetic judgement and may involve evolving up to several hundred intermediate forms. Despite the cliché of computer automation and efficiencies, this is quite often a long and fatiguing process.

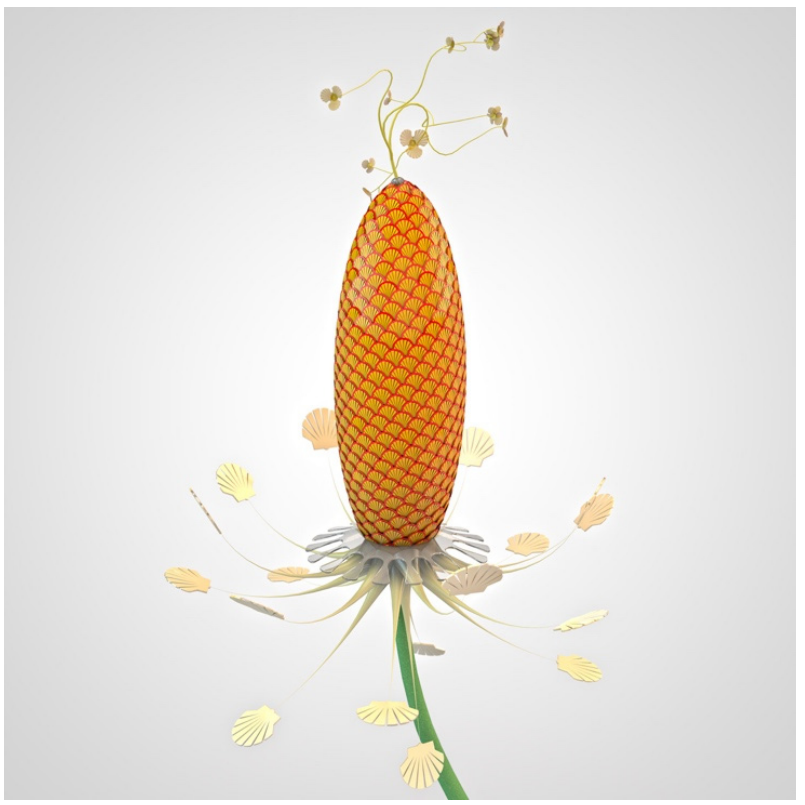

Figure 3: Fifty Sisters: Shell. Plant form grown from the Shell Oil logo.
The unique role of generative systems is illustrated in several ways in Fifty Sisters. The set of rules that define each plant form is described in only a few hundred bytes, but the geometric complexity of the final models is many orders of magnitude larger $\left(\sim 10^{9}\right.$ bytes $)$ illustrating the "database amplification" effects of generative systems.

Secondly, the evolutionary process has introduced many strange and unexpected elements that were not envisaged or explicitly designed (breaking the "Dictum of Descartes") in the software, or components of the original forms. Finally, the images demonstrate the emergent, selforganisation of form that computational generative systems can produce (Figure 3 ).

\section{THE UNKNOWABLE}

The Unknowable is a digital video triptych, developed between 2012-2017. Three portrait format video loops, approximately $10 \mathrm{mins}$ each, depict different plant species in continuous states of decay and reformation (A video excerpt can be found at: https://vimeo.com/113253401). Each plant form slowly disintegrates into its underlying building block - the polygon - and is then reassembled back to its original pristine form, "undoing" the method of its destruction. Like Fifty Sisters, the work is a mediation on our changing relationship with the natural world, now so often experienced indirectly via the digital screen.

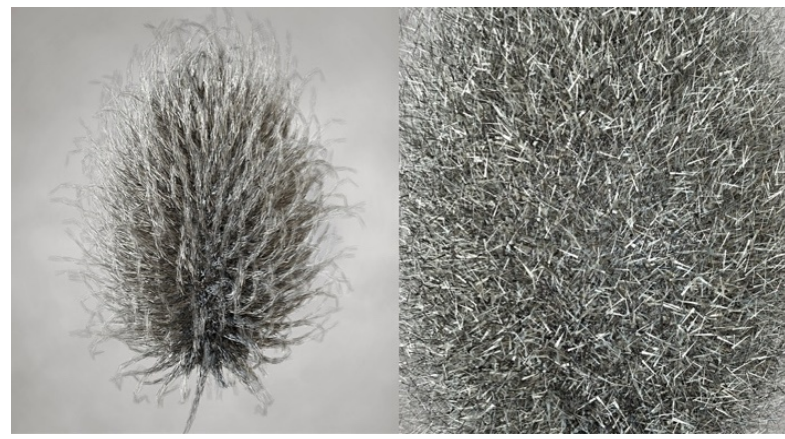

Figure 4: Still frames from "The Unknowable" (detail).

The basic technical mechanisms of production are similar to those used for Fifty Sisters as discussed above, with the exception that these forms are derived from endangered Australian plant species and do not use oil company logos as basic geometric primitives. Aesthetically the work has significant differences, adopting a muted, largely monochrome palette and a darker, more menacing tone that the clean graphic style of Fifty Sisters.

The three screens are used in different ways at various stages of the work. At certain points, they view a continuous visa (Figure 5, top); at other times, they provide different views on the 
construction and disintegration process (Figure 5, bottom).

While the generative process is evident in this work, numerous conceptual, aesthetic and procedural issues that need to be considered in creating a work like this are equally as important and are essential components of my creative practice.
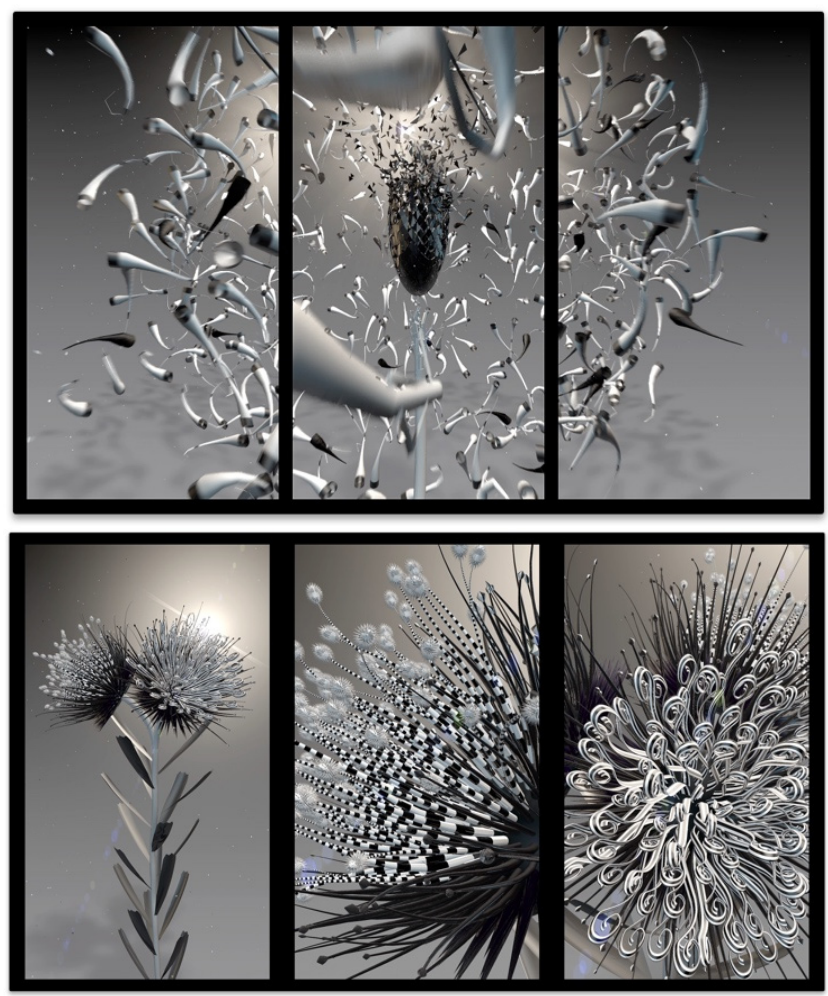

Figure 5: Still images from "The Unknowable".

The Unknowable hints at what I have previously described as the computational sublime: the instilling of simultaneous feelings of pleasure and fear in the viewer of a process created in a computer who's complexity operates at the edges of human comprehension (McCormack \& Dorin 2001).

\section{THEORIES AND CRITIQUES OF GENERATIVE SYSTEMS}

Art that makes use of generative systems has been the subject of analysis and critique, including by the author and colleagues (McCormack et al. 2014). In this section I will briefly outline relevant theories and respond to some of the issues raised in critiques of generative art. For more information, the reader is referred to (McCormack 2003 \& 2013).

\subsection{Algorithmic Genericism}

A common criticism of works employing generative algorithms is that they are generic. Generative artworks are often derived from a common pool of algorithms, such as cellular automata, L-systems,
Voronoi partitioning, reaction-diffusion systems, and so on. These techniques originated in the sciences, not the arts, so understandably they evoke scepticism when introduced as part of an artistic process. Such scepticism is summed up by media theorist Jussi Parikka in a discussion on the use of electronic art made with genetic algorithms (Parikka 2008):

\section{"... if one looks at several of the art pieces made with genetic algorithms, one gets quickly a feeling of not 'nature at work' but a Designer that after a while starts to repeat himself. There seems to be a teleology anyhow incorporated into the supposed forces of nature expressed in genetic algorithms."}

Parikka is also concerned with the "vague characterisation of art" often found in technical writing on this topic and is critical of the references to Art exclusively as a "subjective element", where art-making is understood in pre-modernist terms more as a craft, but with digital tools.

These and similar criticisms can be characterised by three related points:

(i) That works made with similar generative procedures - even by different artists possess a certain generic and repetitive character;

(ii) Works belie a "truth to process" in that they do not exhibit the characteristics or emergent outcomes that the artists' claim the processes used represent;

(iii) Works exclude artistic possibilities due to their technical focus or even fetishism.

Addressing the first point, it is typically the case that similar algorithms produce similar results. Repeatability and determinism are fundamental utilities of computation after all.

There is nothing "generic" in the writing produced by different people using the same word processor software for example, so why would generative art exhibit a repeatability in what it produces that other computational methods do not?

The answer lies in the processual nature of generative systems being central to their conceptualisation as artworks, as opposed to scientific simulations. In terms of my word processor example, this is akin to how one writes prose on a word processor, not the fact that it is written using a word processor. Generative systems emphasise process and a generic process will produce generic work. In considering generative systems in an artistic context, the "Art" is in the process. If that process is derivative or memetic then the work itself will likely share similar properties. 
Both Fifty Sisters and The Unknowable share very similar generative processes, but are differentiated by other factors in the creative process that operate outside the generative system itself. The generative systems on which they are based, while derived from prior research, were uniquely developed as part of an artistic practice. This individuality is further attenuated by the conceptual and aesthetic choices that inform both works' production.

\subsubsection{Truth to Process}

The second point - on "truth to process" - highlights an on-going tension between different worldviews found in art and science. It is also related to debates regarding authenticity and autonomy in generative art (discussed in the next section).

Many generative artists draw inspiration from natural processes, which since the advent of digital computing have been the subject of computational simulation studies (and prior to digital simulation, mathematical modelling). In scientific terms, a simulation is almost always homoeomorphic in its mapping from reality to simulation: in simple terms, it must simplify for expediency, because, like Borges' map (which acquired detail to the point that the map was as detailed as the world it represented), an isomorphic simulation would become the reality it seeks to model. Scientific simulations are generally developed for explanatory purposes not artistic ones.

While in scientific simulations we might look for verification and validation of a model in data from the real world, such activities seem at cross purposes to art. Similarly, considering a process as being true to the simulation rather than true to what the simulation claims to be modelling is equally unexciting.

The various concepts of truth in art have a long and distinguished lineage which make it difficult to adequately consider them in this short paper. Suffice to say that, as with the previous point, any "truth to process" in artistic terms needs to appreciate the authenticity of the generative process as fundamental to the artwork.

\subsubsection{Technical Exclusion}

The final point - that most artistic possibilities are excluded by a technical focus - has been a regular criticism of many generative works. This criticism often stems from the broad use of generative systems across many different cultural forms and commercial activities, not only the "precious bubble" of fine art. Experiments and studies with generative systems are regular parts of scientific and computational discourses. Confusion may arise when such studies are presented as art, or where art is considered primarily as a "subjective element".
As algorithmic thinking and "learning to code" have increasingly become basic literacy issues in schools and universities (including art schools), new generations of creative practitioners accept coding as a significant (but not exclusive) medium for creative expression. New tools for "creative coding" help minimise the technical complexities and engineering origins of computing, making coding more approachable for artists and designers. Moreover, computers and associated information technologies are an increasingly pervasive element of modern culture, and cultural movements such as makerspaces and hacklabs encourage the idea of technical democracy, learning by doing and embodied and situated cognition - modes long practiced in the art world.

In summary, while the criticism of technical exclusion may have been more pervasive in the past when computers were largely inaccessible and more exclusively technically specialised, such criticisms seem less relevant in today's mainstream technological cultures where computer and information technologies are ubiquitous.

\subsection{Agency and Autonomy}

As previously discussed, one of the main attractions of working with generative systems is their capacity for agency and autonomy. Boden provides an extensive examination of autonomy in computer art (Boden 2010, Chapter 9). She distinguishes between two different kinds of autonomy in non-technological contexts and their parallels in computational art:

- physical autonomy such as that exhibited in homeostatic biological systems, and

- mental/intentional autonomy typified by human free-will.

Boden describes how these different kinds of autonomy are at play in generative art. Concepts often used in generative art, such as emergence, agency, autopoesis, homoeostasis and evolutionary adaptation are all underpinned by self-organisation; something Boden views as synonymous with a specific kind of autonomy where "the system's independence is especially strong: it is not merely self-controlled, but also self-generated" (Boden 2010 , p. 180), with the "self" in self-organisation referring to the impersonal components of the system, not the intentional, mental self.

Boden's second form of autonomy is inherently tied to human freedom: something lacking in the autonomy of generative art or other "bottom-up" systems (such as Artificial Life) that rely on computational emergence and self-organisation, and perhaps a cause of confusion regarding "truth to process" as outlined above. This intentional 
autonomy requires concepts from the Theory of Mind, such as intention, desire and belief. There is nothing in principle that dismisses the possibility of artificial creative systems possessing this intentional autonomy, and indeed, this is something extensively explored by Artificial Intelligence research since its inception. If this class of autonomy is dependent on free-will, then any artificially autonomous creative software would need to make the choice to make art, not be explicitly programmed to do so.

Artistic generative systems illuminate the complex relationships between these different kinds of autonomy. Artists work with such systems because they value their ability to exploit autonomous (in the self-organising, homoeostatic sense) processes, while often (incorrectly) ascribing autonomy in the intentional sense to them. Nonetheless, autonomous generative processes result in more aesthetically challenging and interesting artworks over what can be achieved when the computer is used as a mere tool or "slave". They open the possibility for types of expression not possible using other human tools.

\subsubsection{Authorship and Authenticity}

Issues of agency and autonomy also raise other important concepts challenged by generative art: authorship and authenticity. A program that can change itself in response to external stimulus has the potential to learn and adapt (autonomy in the selforganising sense). Hence it can do things that the programmer never anticipated or explicitly designed into the software, including the potential to act creatively, even if this ability was not part of its original programming. The programmer is the author of the program, but the program might be considered the author of what it produces (anecdotally reinforced by the independence of the program from the author). The kind of generalised learning we attribute to ourselves and other species, remains technically elusive for the time being however.

The authenticity of computer and generative art often provokes quite binary responses. Many theorists, critics and philosophers rule out the idea of computer art entirely being authentic because the art was made by a machine, not a person.

\section{CONCLUSIONS}

In working with the generative systems, I have developed to create these works, I see the role of the computer less as a tool and more as a creative partner. The forms produced by the creative processes described continue to surprise me in what they produce. I am often left wondering: how did the computer come up with that?, as some unexpected, but creatively interesting form is thrown out by the generative process. Generative systems have the potential to surprise and delight, to expand your creative thinking and allow you to create designs that outperform their designer.

\section{ACKNOWLEDGEMENTS}

This work was supported by an Australian Research Council Discovery Projects Grant, DP1094064.

\section{REFERENCES}

Ashby, W. R. (1952) Can a Mechanical Chess-Player Outplay its Designer? British Journal of Philosophy, 3(9), 44-57.

Boden, M. A. (2010) Creativity and Art: three roads to surprise. Oxford, UK: Oxford University Press.

Langton, C. G. (1995) Artificial life: an overview. Cambridge, Mass.: MIT Press.

McCormack, J. (1993) Interactive Evolution of LSystem Grammars for Computer Graphics Modelling. In D. Green \& T. Bossomaier (eds.), Complex Systems: from Biology to Computation (pp. 118-130). Amsterdam: ISO Press.

McCormack, J. (2003) Art and the Mirror of Nature. Digital Creativity, 14(1), 3-22.

McCormack, J. (2005) A Developmental Model for Generative Media. In M. Capcarrere, A. A. Freitas, P. J. Bentley, C. G. Johnson, \& J. Timmis (eds.), Advances in Artificial Life (8th European Conference, ECAL 2005). LNAI, volume 3630, pp. 88-97. Berlin; Heidelberg: Springer-Verlag.

McCormack, J. (2013) Aesthetics, Art, Evolution. Paper presented at the EvoMUSART, Vienna, Austria, 3-5 April.

McCormack, J., Bown, O., Dorin, A., McCabe, J., Monro, G., and Whitelaw, M. (2014). Ten Questions Concerning Generative Computer Art. Leonardo, 47(2), 135-141.

McCormack, J. and Dorin, A. (2001) Art, Emergence and the Computational Sublime. Paper presented at the Second Iteration: A Conference on Generative Systems in the Electronic Arts, Melbourne, Australia, 5-7 December.

Parikka, J. (2008) Book review: The Art of Artificial Evolution: A Handbook on Evolutionary Art and Music. Leonardo Reviews.

http://www.leonardo.info/reviews/nov2008/parikka art. $\underline{\mathrm{html}}$

Prusinkiewicz, P. and Lindenmayer, A. (1990) The algorithmic beauty of plants. New York: SpringerVerlag.

Smith, A. R. (1984) Plants, Fractals and Formal Languages. In $\mathrm{H}$. Christiansen (ed.), Computer Graphics (Minneapolis, Minnesota), 18, pp. 1-10. New York: ACM SIGGRAPH. 